\title{
Multi-threaded Geant4 on the Xeon-Phi with Complex High-Energy Physics Geometry
}

\author{
Steven Farrell, Andrea Dotti, Makoto Asai, Paolo Calafiura, Romain Monnard
}

\begin{abstract}
To study the performance of multi-threaded Geant4 for high-energy physics experiments, an application has been developed which generalizes and extends previous work. A highly-complex detector geometry is used for benchmarking on an Intel Xeon Phi coprocessor. In addition, an implementation of parallel I/O based on Intel SCIF and ROOT technologies is incorporated and studied.
\end{abstract}

\section{INTRODUCTION}

$\mathbf{I}$ $\mathrm{N}$ the midst of the multi-core era, the computing models employed by high-energy-physics (HEP) experiments must evolve to embrace the trends of the processor-chip-making industry. As the computing needs of these experimentsparticularly those at the Large Hadron Collider (LHC) grow, adoption of many-core architectures and highly-parallel programming models is essential to prevent degradation in scientific capability.

Simulation of particle interactions is typically a major consumer of CPU resources in HEP experiments. The recent release of a highly performant multi-threaded version of Geant4 [1][2] opens the door for experiments to fully take advantage of highly-parallel technologies.

Intel's many-integrated-core (MIC) processor architecture, known as the Xeon Phi product line, define a platform for highly-parallel applications. Their large number of cores and Linux-based environment make them an attractive compromise between conventional CPUs and general-purpose GPUs. Xeon Phi processors will be appearing in several next-generation supercomputers such as Cori at NERSC.

To prepare for these next-generation supercomputers, a Geant4 application (HepExpMT) has been developed to run multi-threaded HEP particle simulations on the Xeon Phi. The application serves as a realistic demonstrator of the capabilities of this advanced architecture for HEP experiments with complex geometry and parallel writing of particle hit information. It also provides valuable performance measurements for Geant 4 which have already been used to introduce significant improvements in the memory consumption footprint in release (10.1) [3].

\section{MANY-INTEGRATED-CORE PROCESSORS}

Intel's MIC product line, the Xeon Phi, is a powerful processor that can provide good performance for properly optimized

Manuscript received November 23, 2015.

S. Farrell and P. Calafiura are with LBL Lawrence Berkeley National Laboratory, Berkeley, CA 94720 (USA) (corresponding author - telephone: 510-486-4181, email: SFarrell@lbl.gov).

A. Dotti and M. Asai are with SLAC National Accelerator Laboratory, Menlo Park, CA 94025 (USA).

R. Monnard is with HES-SO Haute Ecole Spécialisée de Suisse Occidentale, Fribourg (Switzerland). applications. The current generation, known as Knights Corner (KNC), is a coprocessor chip that functions alongside a traditional CPU and supports both offload and native programming models. It has more than 50 cores with 512-bit advanced vector instructions (AVX) running a simplified Linux OS.

The KNC coprocessor doesn't have a hard disk and has a limited amount of RAM (6-16 GB). A performant communication mechanism between host and coprocessor is thus essential for applications that produce a significant amount of output. The Intel Symmetric Communications Interface (SCIF) library serves this purpose, providing high-performance direct communication and remote memory access (RMA) operations designed to exploit the full bandwidth capability of the PCI express bus which connects the host and the coprocessor.

The next generation Xeon Phi, known as Knights Landing (KNL), will provide significant improvements in compute power and usability. It will be a standalone fully $\mathrm{x} 86$ compatible processor with 72 cores, each one delivering three times the performance of a KNC core. The KNL also comes with 8-16 GB of high-bandwidth memory (MCDRAM) and support for up to $384 \mathrm{~GB}$ of regular RAM.

Xeon Phi processors already play a significant role in current and future supercomputers. KNC chips are currently used in machines such as Tianhe-2 at the National Supercomputer Center in Guangzhou and Stampede at the Texas Advanced Computing Center (respectively \#1 and \#8 on the current TOP500 list). Supercomputers that are planned to use KNL chips include Cori at NERSC and Theta at Argonne National Lab.

\section{Multi-Threaded Geant4}

Support for multi-threading in Geant4 is available since release version 10.0 (December 2013). The goal of the design is to make efficient use of multi-core processors and reduce the memory footprint with respect to a sequential application. The multi-threaded design is based on a master-worker model and the POSIX standard. Each worker thread is responsible for simulating one or more full events, thus implementing eventlevel parallelism. The master thread is responsible for managing shared data structures and initializing the worker threads. Threads are independent and require minimal synchronization, which results in very performant scaling up to the number of physical cores on a chip.

\section{ROOT I/O}

The ROOT [4] data analysis framework provides functionality for writing out HEP event information in a specialized data format ("ROOT files"). HEP simulation applications need 
to write out information describing particle energy deposits in sensitive detector elements ("hits") in an output ROOT file. There are multiple ways to implement output writing when running events concurrently. The simplest approach is to have each worker process events independently and write to separate files on disk which can be merged at the end of processing or during the analysis stage. ROOT however provides some functionality for writing data to a single output file in parallel. A specialized type of file called TParallelMergingFile uses sockets to connect clients to a server via TCP which does the merging of outputs into a single file.

On a KNC coprocessor it is necessary to ship the particle hits output data to the host to write to disk because of the limited RAM budget on the card. The TParallelMergingFile implementation can be used for this, but it has been shown [5] that socket-based communication incurs significant overhead on the Xeon Phi. Instead, communication based on the Intel SCIF library is able to achieve data bandwidth much closer to the theoretical maximum bandwidth of the PCI express bus [5].

In order to utilize the high-performance capability of Intel SCIF, a new backend was written for ROOT. A new ROOT file implementation, TSCIFFile, allows to use this backend to send data in chunks to the host CPU where a merging server collects them and merges them to disk [5].

\section{HEPEXPMT: AN ADVANCED MULTI-THREADED GEANT4 BENCHMARK AND DEMONSTRATOR}

HepExpMT is an evolution and upgrade of an existing multi-threaded application ("ParFullCMS" [6], [7]) developed by Geant 4 for testing code correctness with HEP geometry. ParFullCMS uses the Xerces-C library [8] and the Geant4 GDML [9] parser to build a simplified CMS detector geometry provided at run-time via a GDML file. A uniform magnetic field is applied to the setup and single particles of a given energy are shot in a random direction and simulated with the Geant4 physics engine.

The new application has been upgraded for increased complexity and realism, and also made general enough to run any GDML detector simulation. For performance studies, the ATLAS detector geometry is used because its very large number of geometrical elements, $\mathcal{O}\left(10^{6}\right)$, gives a very challenging setup to test multi-threading capabilities of Geant4. The uniform magnetic field along the $z$ axis was not changed, since for these testing purposed a uniform field is considered sufficient. The application has been generalized to allow for control of the primary generation via macro commands to test different aspects of the physics engine.

The implementation of SCIF-based I/O described in Section [V] has been included in HepExpMT to write particle hits output data in parallel. No sensitive detectors have been implemented in this initial version of the code, but energy deposits at every particle tracking step are converted into hit data to write out for each simulated event. The data is sent regularly to the host via SCIF RMA where a server process merges the results to a file on local disk.

HepExpMT has been bundled with its support scripts and external libraries in a standalone package. It is now possible to distribute, compile, and run the application on different architectures and linux systems without the need of any external dependency on pre-installed software. The package will be made public in the near future to allow for users to perform testing of Geant 4 and hardware performance evaluations with custom geometries.

The development and testing of HepExpMT has helped to uncover limitations and bugs in the underlying software packages, thereby providing valuable feedback to respective developers. A limitation was identified and patched in the Xerces-C implementation for extremely large XML files (like the ATLAS GDML). A couple of Geant4 bugs related to GDML writing and parsing were fixed. Finally, the results of memory consumption measurements prompted significant improvements to the memory handling in the Geant 4 physics code.

\section{PERFormancE MEASUREMENTS}

The performance of the HepExpMT Geant4 application was measured on a 5110p Knights Corner Xeon Phi with 60 cores and $8 \mathrm{~GB}$ of RAM. Two different GDML files based on the ATLAS detector were used as input. The "full-ATLAS" GDML has full detail of the detector except for the ATLAS hadronic end-cap calorimeter which cannot be represented in GDML form. A second, simpler version describing only the ATLAS inner detector (the "ID-ATLAS" GDML) is used for the measurements with output writing. A uniform field was simulated at $4 \mathrm{~T}$, and a fixed number of pions were fired from the interaction point in random directions with $50 \mathrm{GeV}$ of momentum.

The first set of measurements use the full-ATLAS detector without I/O to test the scalability of Geant 4 with the most complex detector setup. Figure 1 shows how the event processing rate (throughput) scales with the number of threads. The throughput shows nearly perfect scaling up to the number of cores on the chip (60), showing that the Geant4 multithreading design is very efficient and introduces minimal overhead and contention. The throughput continues to increase in the hyper-threading regime up to the maximum possible 4 threads per core (240 threads). Figure 2 shows how the resident memory of the application scales with the number of threads. The linear increase is expected and shows that each thread contributes roughly the same amount of memory. The coprocessor runs out of memory when running 240 threads, which is an unfortunate consequence of the limited RAM budget ( $8 \mathrm{~GB}$ ) of the KNC card ${ }^{1}$ Finally, the memory consumption during the runtime of the application is show in Figure 3 for several different threading configurations. The plot shows a very long plateau from the parsing and processing of the GDML input, and a rise and plateau during the event loop. This shape is due to the lazy initialization of memory in the Geant4 physics code.

To measure the impact of parallel I/O on the application performance, the simpler ID-ATLAS GDML is used. The output data size per event is held fixed at $4 \mathrm{MB}$ when

\footnotetext{
${ }^{1}$ The data-files needed to run the application are copied to the RAM of the card, no use of NSF has been employed for this study.
} 


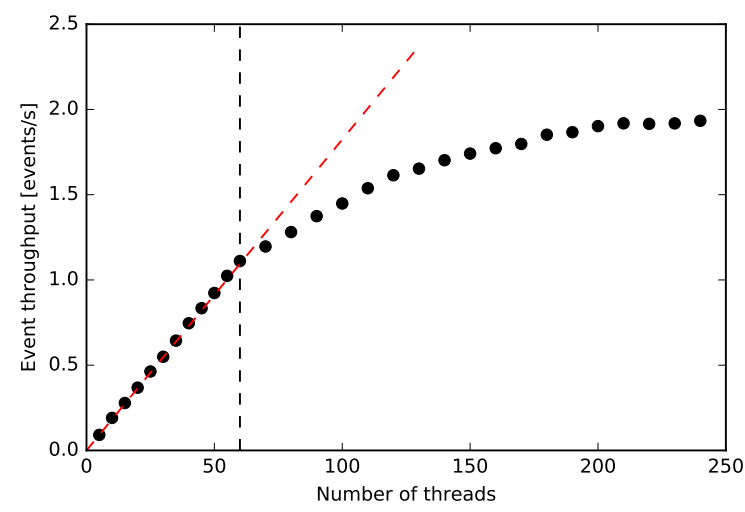

Fig. 1. Event processing throughput of the HepExpMT application on the Xeon Phi coprocessor as a function of the number of threads. The total number of events processed is chosen as 100 times the number of threads. Nearly perfect scaling is observed up to the number of physics cores on the chip (60, represented by the dashed vertical line), with increasing rate up to the maximum 4 threads per core.

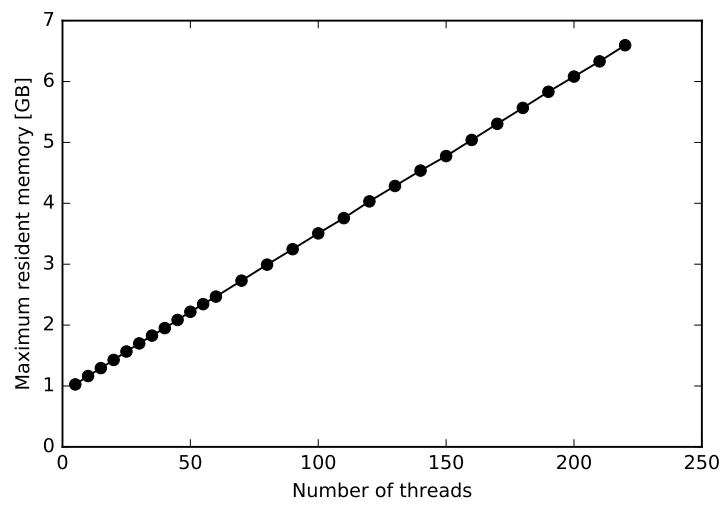

Fig. 2. Resident memory of the HepExpMT application on the Xeon Phi coprocessor as a function of the number of threads. The number of events processed is 100 times the number of threads.

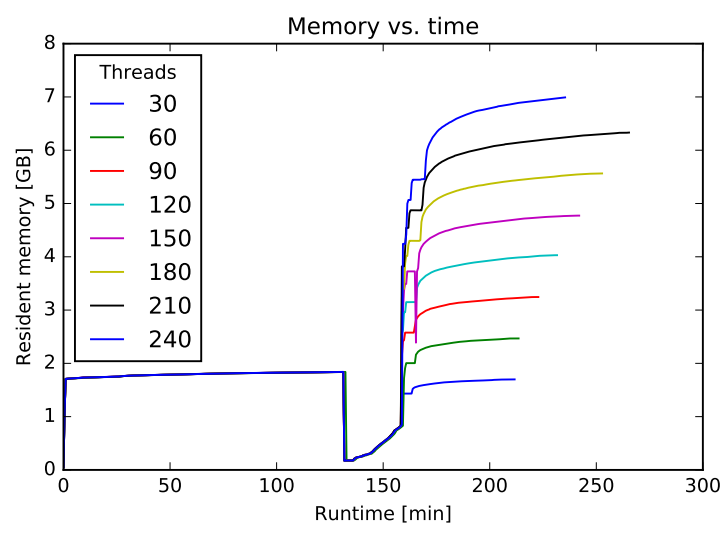

Fig. 3. Resident memory of the HepExpMT application on the Xeon Phi as a function of application run time for several thread configurations. The number of events processed is 100 times the number of threads. The long initial plateau is the GDML parsing and detector building. The second rise and plateau is the event loop processing.

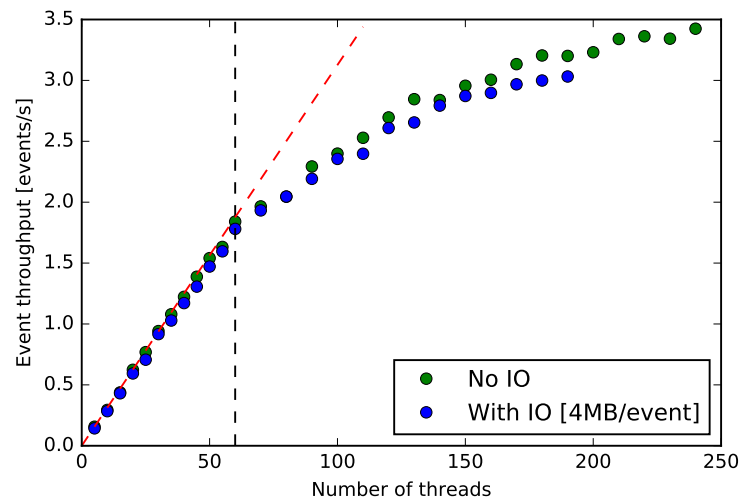

Fig. 4. Event processing throughput of the HepExpMT application on the Xeon Phi coprocessor as a function of the number of threads, shown with and without $\mathrm{I} / \mathrm{O}$. The number of events processed is 50 times the number of threads. No significant impact is observed on throughput due to the I/O.

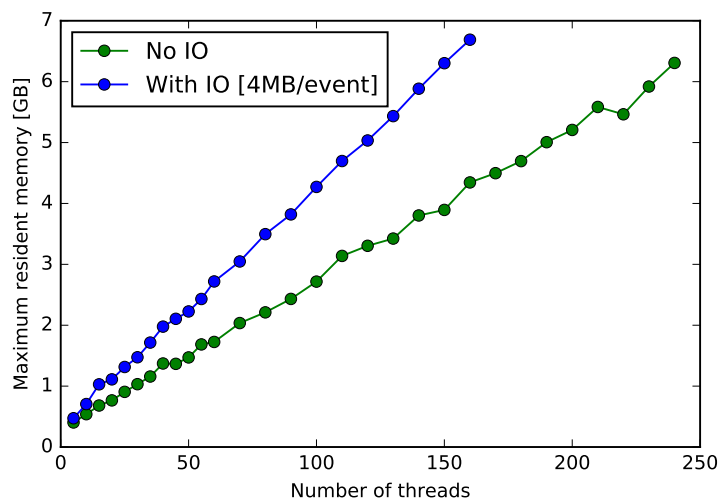

Fig. 5. Resident memory of the HepExpMT application on the Xeon Phi coprocessor as a function of the number of threads, shown with and without $\mathrm{I} / \mathrm{O}$. The number of events processed is 50 times the number of threads.

writing with TSCIFFile. Figure 4 shows the event throughput comparison with and without $\mathrm{I} / \mathrm{O}$. There is little to no impact observed from the output writing, which means there are no communication bottlenecks and no significant overhead in the I/O layer. Figure 5 shows the linearity of the memory scaling with and without I/O. The I/O layer increases the memory consumption by a fixed amount per thread, which causes jobs to be aborted for fewer number of threads. The memory consumption during the runtime is shown for this configuration without I/O in Figure 6 and with I/O in Figure 7. These plots show that the upward shift in memory consumption of the I/O is fairly flat in time during the event loop.

\section{CONCLUSIONS}

This work demonstrates the feasibility of using Xeon Phi for multi-threaded Geant4 simulations with complex HEP geometry via a new advanced benchmark application, HepExpMT. Measurements of event throughput and memory consumption show that Geant 4 performs very well with a large number of threads and a limited memory budget, making it well suited for the MIC architecture. Writing of event data in parallel using a 


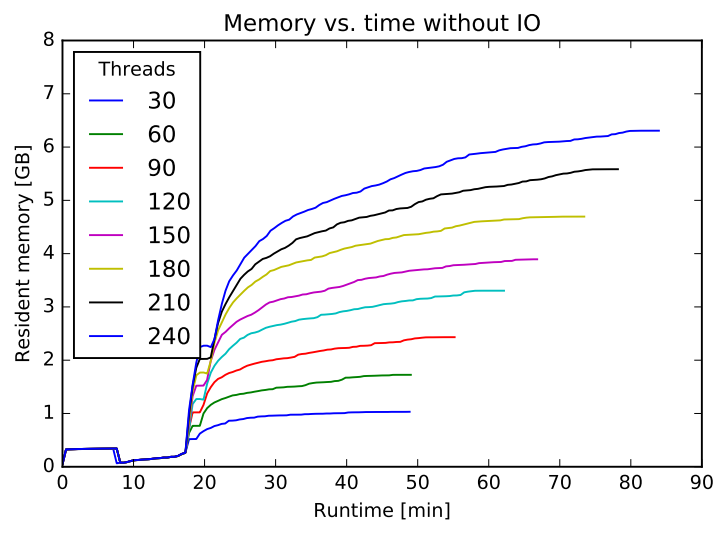

Fig. 6. Resident memory of the HepExpMT application with the ID-ATLAS GDML and no I/O on the Xeon Phi as a function of application run time for several thread configurations and no I/O. The number of events processed is 50 times the number of threads.

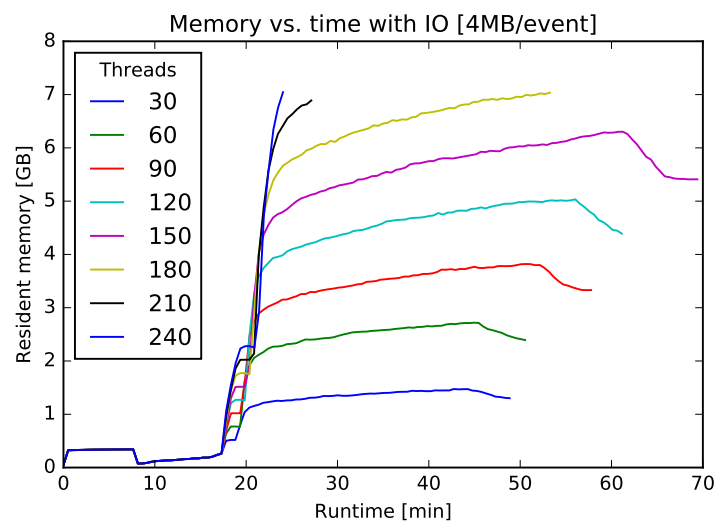

Fig. 7. Resident memory of the HepExpMT application with the IDATLAS GDML with parallel output writing on the Xeon Phi as a function of application run time for several thread configurations. The number of events processed is 50 times the number of threads.

SCIF-backend for ROOT is shown to perform well and have no significant impact on event throughput.

This work serves as a valuable learning experience and stepping stone to prepare HEP experiments for the next-generation Xeon-Phi-based supercomputers such as Cori. These new machines will be built with the KNL generation of Xeon Phi, though, which has significant design updates with respect to the KNC architecture used for these results. In particular, the difficulties with the tight memory constraints will be relaxed thanks to the increased memory capacity of the KNL cards. Also, the I/O implementation will likely change because the KNL cards are self-hosted and can write directly to hard disks or shared filesystems. However, the parallel I/O mechanisms used in these results will still be the preferable way to save event data, so the overall scheme may look similar. New studies will need to be performed when the new Xeon Phi cards become available.

\section{REFERENCES}

[1] S. Agostinelli et al., "Geant4-a simulation toolkit," Nuclear Instruments and Methods in Physics Research, Section A: Accelerators, Spectrometers, Detectors and Associated Equipment, vol. 506, no. 3, pp. 250 - 303, 2003.

[2] J. Allison et al., "Geant4 developments and applications," Nuclear Science, IEEE Transactions on, vol. 53, no. 1, pp. 270-278, 2006.

[3] M. Asai et al., "Geant4 version 10 series," in Joint International Conference on Mathematics and Computation, Supercomputing in Nuclear Applications and the Monte Carlo Methods, 2015.

[4] R. Brun and F. Rademakers, "ROOT: An object oriented data analysis framework," Nucl. Instrum. Meth., vol. A389, pp. 81-86, 1997.

[5] R. Monnard, "Concurrent I/O from Xeon Phi accelerator cards," Master's thesis, Haute École Spécialisée de Suisse Occidentale de Fribourg, Switzerland, 2015.

[6] S. Ahn et al., "Geant4-MT: bringing multi-threading into Geant4 production," in Joint International Conference on Supercomputing in Nuclear Applications and Monte Carlo, vol. 2013, 2013.

[7] G. Cosmo, "Geant4 - towards major release 10," Journal of Physics: Conference Series, vol. 513, no. 2, p. 022005, 2014. [Online]. Available: http://stacks.iop.org/1742-6596/513/i=2/a=022005

[8] [Online]. Available: http://xerces.apache.org/xerces-c/

[9] R. Chytracek et al., "Geometry description markup language for physics simulation and analysis applications," IEEE Trans. Nucl. Sci., vol. 53, no. 5 , pp. 2892-2896, 2006. 